\title{
A utilização do mapeamento cruzado na peseuisa de enfermagem: uma revisão da literatura*
}

\author{
The use of cross-mapping in nursing researches: a literature review \\ El uso del mapeo cruzado en las investigaciones de enfermería: una revisión de la literatura
}

\section{Fernanda de Oliveira Lima Nonino', Anamaria Alves Napoleão', Emília Campos de Carvalho", José Fernando Petrilli Filho'}

'Universidade Federal de São Carlos. Departamento de Enfermagem. São Carlos, SP

"Universidade de São Paulo. Escola de Enfermagem de Ribeirão Preto. Ribeirão Preto, SP

Submissão: 08/07/2008

Aprovação: 18/1 1/2008

\section{RESUMO}

Trata-se de um estudo de revisão de literatura cujo objetivo foi identificar e analisar a utilização do mapeamento cruzado nas pesQuisas em enfermagem. Identificou-se que as pesquisas que utilizaram o mapeamento cruzado na enfermagem possuem relação com a criação dos sistemas de classificação e com a perspectiva do uso da informática nos serviços de saúde. Em relação aos países Que realizaram pesquisas utilizando o mapeamento cruzado aparecem os Estados Unidos com 11 estudos, seguido do Brasil com 6 estudos, Holanda com 3 estudos e outros países da Europa e Ásia com I estudo cada. O mapeamento cruzado é uma ferramenta útil em estudos sobre linguagens padronizadas em enfermagem e pode favorecer o uso dos sistemas de classificação nos diferentes contextos.

Descritores: Enfermagem; Classificação; Pesquisa em enfermagem.

\section{ABSTRACT}

This literature review aims to identify and to analyze the use of cross mapping in nursing researches. It was identified that researches that used cross mapping in nursing are related to the creation of classification systems and also with the prospect of the use of computers in health services. The United States performed 1 I studies using the cross mapping followed by Brazil with 6 studies, Netherlands with 3 studies and other countries in Europe and Asia with I study. The cross mapping is a useful tool in studies on the standardized languages in nursing and can promote the use of the classification systems in different contexts.

Descriptors: Nursing; Classification; Nursing research.

\section{RESUMEN}

Este estudio de revisión de la literatura tiene como objetivo identificar y analizar el mapeo cruzado en investigaciones en enfermería. Se identificó Que las investigaciones con mapeo cruzado en enfermería poseen relación con la creación de los sistemas de clasificación y también con la perspectiva del uso de la informática en los servicios de salud. Los países Que realizaron investigaciones utilizando el mapeo cruzado son Estados Unidos con 11 investigaciones, Brasil con 6 investigaciones, Holanda con 3 investigaciones y otros países de Europa y Asia con 1 investigación cada uno. El mapeo cruzado es una herramienta usada en estudios sobre los lenguajes estandarizados en enfermería y pueden favorecer el uso de los sistemas de clasificación en los distintos contextos.

Descriptores: Enfermería; Clasificación; Investigación en enfermería. 


\section{INTRODUÇÃO}

As linguagens padronizadas na enfermagem têm sido desenvolvidas e divulgadas, a exemplo dos sistemas de classificação de diagnósticos de enfermagem da North American Nursing Diagnosis Association (NANDA), de classificação de intervenções de enfermagem (NIC), de classificação dos resultados de enfermagem (NOC) e da classificação internacional para a prática da enfermagem (CIPE).

Autores apontam Que estas linguagens possuem um papel fundamental em desenvolver e definir os fenômenos e ações da enfermagem ${ }^{(1)}$, assim como descrever claramente as contribuições da enfermagem no cenário de cuidados à saúde ${ }^{(2)}$.

Apesar de serem importantes, sua utilização ainda é incipiente na realidade brasileira, onde se observam registros realizados sem a adoção de um sistema uniformizado com base em uma classificação(3).

Ao comentar sobre os benefícios da utilização de um sistema de classificação de intervenções de enfermagem como a NIC, autores identificam a possibilidade de nomear as ações realizadas, facilitar o registro dessas ações, reforçar a importância de ações efetivamente realizadas no cotidiano, conferir maior visibilidade ao trabalho do enfermeiro e ainda identificar atividades não realizadas e discutir os aspectos relacionados à sua não realização, como os legais, estruturais, condições de trabalho, entre outros ${ }^{(4)}$.

No contexto do desenvolvimento de linguagens padronizadas da enfermagem, o "cross-mapping", traduzido como mapeamento cruzado, pode ser identificado como uma ferramenta Que permite a comparação destas com outras linguagens, tais como aquelas Que são utilizadas no cotidiano dos serviços ou ainda as de outros sistemas de classificação já existentes, entre outras.

Autores Que utilizaram o mapeamento cruzado em seus estudos exemplificam mostrando os termos a serem comparados dispostos paralelamente ${ }^{(3)}$, explicam as regras adotadas para a realização do mapeamento a partir de estudiosos e as adaptações realizadas a partir das regras ${ }^{(4)}$.

O mapeamento cruzado foi descrito como uma ferramenta útil na realidade brasileira no Que se refere aos serviços de saúde Que planejam implantar os sistemas de classificação de Enfermagem já existentes e já possuem um sistema próprio de organização para as informações de enfermagem, uma vez Que permite demonstrar Que os dados já existentes podem ser mapeados nas classificações de Enfermagem e adaptados para a linguagem padronizada ${ }^{(3)}$.

Diante da relevância da utilização das linguagens padronizadas na enfermagem e do mapeamento cruzado como uma importante ferramenta nesse contexto julgou-se pertinente a realização de um estudo Que identificasse como este recurso tem sido utilizado por pesquisadores da enfermagem. Sendo assim, o objetivo proposto foi identificar e analisar a utilização do mapeamento cruzado na literatura científica de enfermagem.

\section{METODOLOGIA}

Trata-se de um estudo de revisão da literatura realizado no ano de 2007. O levantamento foi realizado nos meses de outubro e novembro de 2007. As bases de dados utilizadas foram Literatura da América Latina e Caribe - LILACS, no sítio da Biblioteca Virtual em Saúde - BIREME (www.bireme.br) e Medical Literature on Line - MEDLINE, no sítio da PUBMED (http://www.ncbi.nlm.nih.gov/ sites/entrez? db= pubmed).

Os termos combinados utilizados na base de dados MEDLINE foram: cross, mapping, nursing e também mapping, nursing, languages. Os termos combinados utilizados na base LILACS, foram: mapeamento, cruzado, enfermagem e também mapeamento, enfermagem.

Foram incluídos no presente estudo artigos científicos Que tratavam da utilização de mapeamento cruzado na enfermagem cujos resumos estavam disponíveis em inglês, português ou espanhol nas bases de dados e apresentavam objetivos, metodologia, resultados e conclusões. Caso o manuscrito estivesse disponível na íntegra via eletrônica ou em acervo particular, não necessariamente o resumo necessitaria apresentar os itens acima descritos, uma vez Que as autoras verificariam esses dados no corpo do texto.

Na base de dados MEDLINE, com a combinação dos termos mapping, nursing, languages obteve-se 34 referências. A partir da leitura dos títulos e/ou resumos e observados os critérios de inclusão, foram selecionados um total de 21 resumos.

Nesta mesma base, com a combinação dos termos cross, mapping, nursing foi obtido um total de $2 \mathrm{I}$ referências. A partir da leitura dos títulos e/ou resumos e observados os critérios de inclusão, foram selecionados 9 resumos. Destes, 7 se repetiam com o levantamento anteriormente citado. Assim, 2 resumos foram incluídos para análise. Desta forma, na base de dados MEDLINE foram selecionados 23 resumos para análise.

Na base de dados LILACS, com a combinação dos termos mapeamento e enfermagem obteve-se 14 referências. Observados os critérios de inclusão, 5 resumos foram selecionados. Com o uso dos termos mapeamento, cruzado e enfermagem foram levantadas 3 referências, todas repetidas. Desta forma, na base de dados LILACS foram selecionados 5 resumos, sendo que 2 foram levantados também na MEDLINE e considerados nesta base para fins de distribuição no presente trabalho.

No total, 26 resumos foram selecionados nas bases de dados consultadas.

Após identificação do número de trabalhos inclúdos, foram analisados e fichados os resumos. Para fins de fichamento os dados foram organizados da seguinte forma: base de dados, número de apresentação do artigo na base de dados, ano de publicação, objetivos, metodologia e conclusões.

O procedimento de busca das referências, assim como o preparo dos fichamentos foram adaptados de acordo com as recomendações apresentadas por Polit, Beck e Hungler ${ }^{(5)}$ para preparação de revisões da literatura. $\mathrm{Na}$ análise e integração do material, os trabalhos selecionados foram agrupados, Quando pertinente, de acordo com a abordagem realizada, a partir da leitura dos objetivos e da identificação dos procedimentos realizados pelos autores.

\section{RESULTADOS E DISCUSSÃO}

Os resultados estão apresentados em uma tabela e dois gráficos, conforme consta a seguir.

Na Tabela I foram distribuídos os estudos de acordo com a abordagem e base de dados onde foram identificados. 
Tabela 1. Distribuição dos estudos analisados de acordo com a abordagem e base de dados em Que foram selecionados. 2007.

\begin{tabular}{|c|c|c|c|}
\hline Categoria & $\begin{array}{l}\text { MEDLINE } \\
n\end{array}$ & $\begin{array}{c}\text { LILACS } \\
n\end{array}$ & $\begin{array}{c}\text { TOTAL } \\
n\end{array}$ \\
\hline $\begin{array}{l}\text { Mapeamento para comparação de linguagens não padronizadas com sistemas de classificação ou } \\
\text { terminologias padronizadas }\end{array}$ & 7 & 2 & 9 \\
\hline $\begin{array}{l}\text { Mapeamento para determinar a correspondência entre diferentes linguagens padronizadas de } \\
\text { enfermagem }\end{array}$ & 6 & - & 6 \\
\hline $\begin{array}{l}\text { Mapeamento para comparar outras terminologias padronizadas com terminologias padronizadas } \\
\text { de enfermagem }\end{array}$ & 3 & - & 3 \\
\hline Descrição do processo de mapeamento (métodos, regras) ou de validação do mapeamento & 2 & 1 & 3 \\
\hline $\begin{array}{l}\text { Mapeamento de termos para criação de terminologia específica para determinado grupo de } \\
\text { pacientes a partir da CIPE }\end{array}$ & 1 & - & 1 \\
\hline $\begin{array}{l}\text { Mapeamento entre base de dados clínicos computadorizada com variáveis de pesquisa em } \\
\text { enfermagem }\end{array}$ & 1 & - & 1 \\
\hline $\begin{array}{l}\text { Mapeamento de terminologias de enfermagem não especificadas por meio de um sistema } \\
\text { padronizado de integração de terminologias em prontuários eletrônicos }\end{array}$ & 1 & - & 1 \\
\hline $\begin{array}{l}\text { Mapeamento entre termos para designar sintomas usados nas fichas de pacientes da oncologia } \\
\text { com termos de uma base de dados específica }\end{array}$ & 1 & - & 1 \\
\hline $\begin{array}{l}\text { Descrição da CIPE como terminologia Que pode facilitar a comparação de linguagens de } \\
\text { enfermagem por meio de mapeamento cruzado }\end{array}$ & 1 & - & 1 \\
\hline Total & 23 & 3 & 26 \\
\hline
\end{tabular}

Observa-se a predominância de estudos Que mapearam linguagens não padronizadas a linguagens padronizadas com 9 estudos no total ${ }^{(2,6-13)}$. Como exemplos podem ser citados dois estudos norte-americanos publicados em 1997 Que mapearam intervenções de enfermagem não padronizadas de bases de dados internas de hospitais às intervenções da $\mathrm{NIC}^{(2,9)}$; estudo norteamericano publicado em 2004 no Qual os autores, após criarem um modelo estrutural de informações usadas em cada etapa do processo de enfermagem, mapearam estas informações ao Modelo de Referência de Informação de uma organização internacional para o desenvolvimento de padrões na área da saúde - o Health Level 7 (HL7) Reference Information Model (RIM), visando examinar como representá-las ${ }^{(10)}$; estudo brasileiro publicado em 2002 Que mapeou termos usados em registros clínicos de pacientes com a CIPE ${ }^{(1)}$ e ainda estudo brasileiro publicado em 2006 Que objetivou identificar fenômenos de enfermagem durante visitas realizadas pela enfermagem por meio de mapeamento de termos identificados em 52 anotações de enfermagem das visitas com termos da CIPE(8).

A segunda abordagem mais identificada referiu-se à utilização do mapeamento cruzado para comparação de linguagens padronizadas de enfermagem entre si. Foram identificados 6 estudos Que faziam esta abordagem ${ }^{(1,14-18)}$. Como exemplos de estudos realizados cita-se um estudo brasileiro publicado no ano de 2003 , em Que os termos que designavam ações de enfermagem do projeto intitulado Classificação Internacional para a Prática de Enfermagem em Saúde Coletiva - CIPESC foram acessados com base nas intervenções de enfermagem estabelecidas pelo Conselho Internacional de Enfermagem e mapeados às intervenções da $\mathrm{NIC}^{(15)}$ e estudo coreano no ano de 2002 em Que os autores mapearam a CIPE com a NANDA, Home Health Care Classification (HHCC), Omaha System e NIC ${ }^{(1)}$.

Uma outra abordagem identificada em 3 estudos foi o mapeamento de outras terminologias padronizadas com sistemas de classificação de enfermagem ${ }^{(19-21)}$. Como exemplo cita-se um estudo norte americano realizado em 2007, cujo propósito foi validar o mapeamento cruzado entre a NOC e a Systematized Nomenclature of Medicine Clinical Terms - SNOMED ${ }^{(21)}$.

Uma Quarta abordagem foi identificada em 3 estudos Que discorreram sobre o processo de mapeamento cruzado Quanto aos seus métodos e regras ${ }^{(3,22-23)}$. Nessa categoria cita-se como exemplo um estudo brasileiro publicado em 2005 que apresenta e descreve o mapeamento cruzado, designando-o como um método ${ }^{(3)}$ e um estudo norte americano Que apresenta a síntese de métodos, regras e aspectos para mapeamento de linguagem padronizada de intervenções de enfermagem ${ }^{(23)}$.

Foram ainda identificadas 5 abordagens, conforme descrito na Tabela I, em 5 diferentes estudos ${ }^{(24-28)}$. Inclui-se um estudo alemão realizado em 2006 em Que os autores apresentam como objetivo a construção de um sub-sistema de termos da CIPE para pacientes da oncologia ${ }^{(24)}$; um estudo norte americano realizado em 1996 em Que os autores discorrem sobre as estratégias para uso de uma base de dados clínicos computadorizada para a pesquisa em enfermagem $^{(25)}$; um estudo Holandês realizado em 2004 em Que o autor discorre sobre o mapeamento de terminologias de enfermagem a um sistema padronizado de integração de terminologias em prontuários eletrônicos - ISO ${ }^{(26)}$; um estudo norueguês realizado em 2006 em Que os autores extraíram termos utilizados para designar sintomas de 25 fichas de pacientes da oncologia e os mapearam com termos de um sistema específico para a oncologia ${ }^{(27)}$; e um estudo realizado na Suíça em 2006 em Que os autores relatam a participação na CIPE e descrevem esta terminologia como facilitadora da comparação de linguagens em enfermagem por meio do mapeamento cruzado ${ }^{(28)}$.

Em relação ao ano de publicação e base de dados utilizada, os dados estão apresentados na Figura 1.

Observa-se, no período de 1996 até 1998, um total de 5 


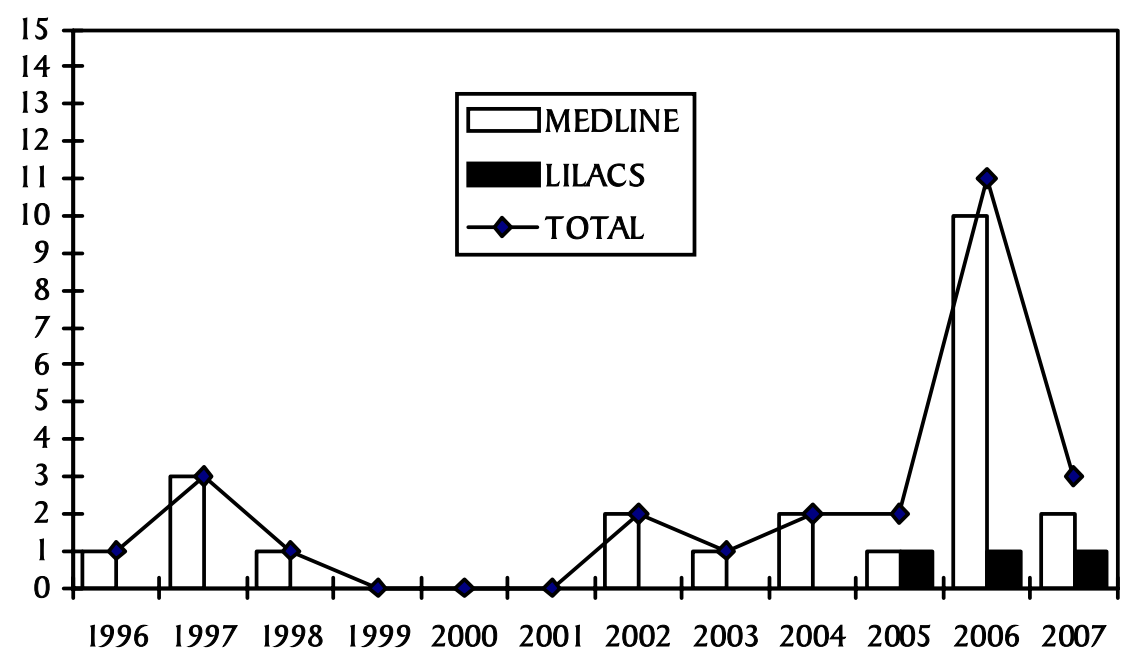

Figura 1. Distribuição dos estudos da enfermagem Que usaram o mapeamento cruzado em relação ao ano de publicação e base de dados consultada. 2007.

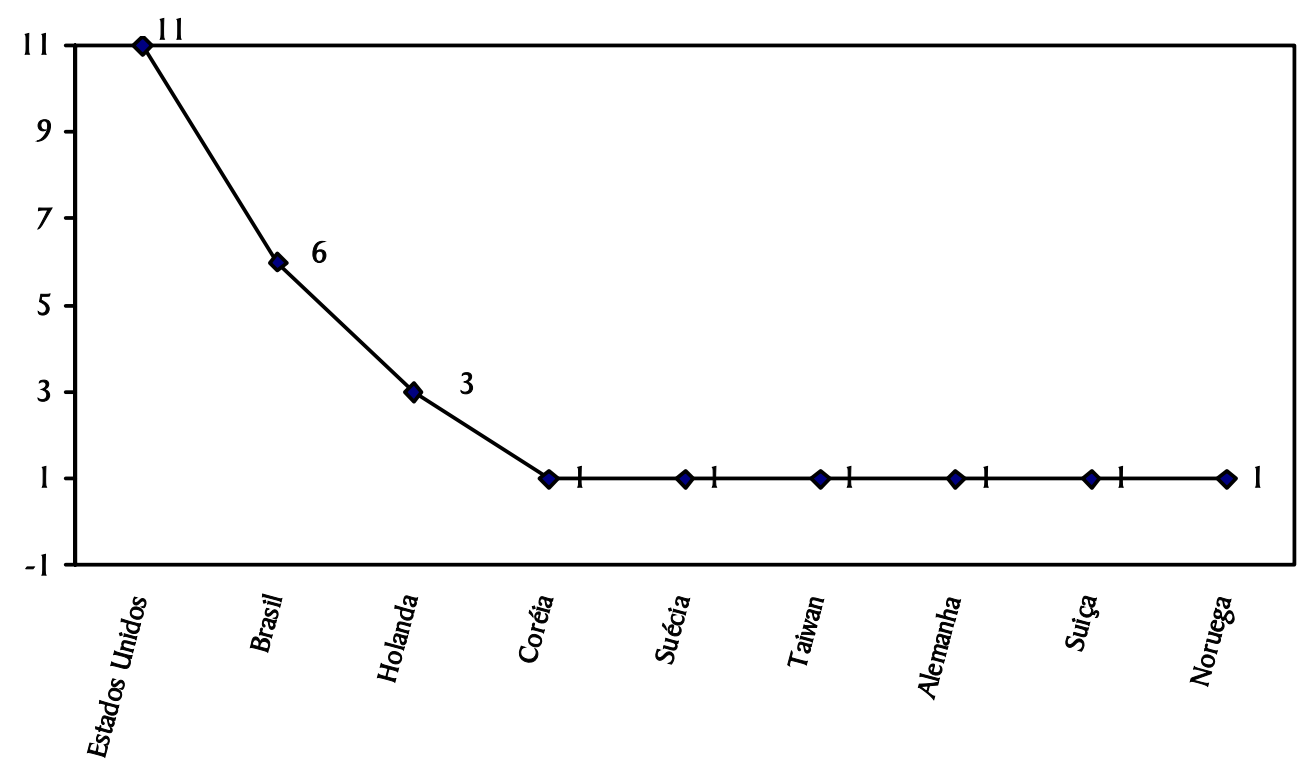

Figura 2. Distribuição de estudos da enfermagem Que utilizaram o mapeamento cruzado segundo o país de origem. 2007.

estudos publicados e um intervalo de 1999 até 2001 em Que nenhum estudo consta nas bases de dados consultadas. Já a partir de 2002 até 2007, 21 estudos foram levantados, sendo Que o ano de 2006 diferencia-se consideravelmente com 11 estudos. Seis dos estudos levantados foram realizados no Brasil, a partir do ano de 2002.

Constata-se que a tendência à utilização do mapeamento cruzado nas pesQuisas de enfermagem possui relação com a criação dos sistemas de classificação e também com a perspectiva do uso da informática nos serviços de saúde, pois a maioria dos estudos levantados envolveu comparações feitas a partir dos sistemas de classificação ou linguagens padronizadas já existentes, inclusive aquelas Que não são específicas da enfermagem, como a
Systematized Nomenclature of Medicine - Clinical Terms (SNOMED) e Logical Observation Identifiers Names and Codes (LOINC).

Em relação ao uso da informática e dos sistemas de classificação na enfermagem autores afirmam Que, pelo fato da documentação dos cuidados de enfermagem estar se tornando cada vez mais informatizada, sistemas de classificação como a NANDA, NIC e NOC podem oferecer à enfermagem elementos dos dados clínicos para um registro automatizado do paciente ${ }^{(29)}$.

Quanto ao país de origem, os dados estão apresentados no Figura 2.

Foram identificados I I estudos realizados nos Estados Unidos. Vale salientar Que este país desenvolve vários sistemas de 
classificação divulgados mundialmente como é o exemplo da NANDA, NIC, NOC, Omaha System, entre outros. O Brasil apareceu em segundo lugar com 6 estudos publicados, indicando uma tendência ao uso dos sistemas de classificação. Em terceiro lugar, a Holanda aparece com 3 estudos e outros 4 países europeus apresentaram I estudo cada, indicando também a tendência ao uso dos sistemas de classificação na Europa. Entre os países asiáticos a Coréia e Taiwan apresentaram I estudo cada.

Em relação aos sistemas de classificação de enfermagem mencionados nos estudos, a CIPE e a NIC aparecem entre os mais citados, indicando Que estas linguagens despertam interesse e podem oferecer uma grande possibilidade de estudos para a padronização dos termos utilizados na enfermagem.

Os resultados do presente estudo sinalizam para a tendência à realização de comparações de linguagens utilizadas na enfermagem, especialmente a partir do surgimento dos sistemas de classificação e da utilização de bases eletrônicas de dados nos diferentes contextos. O mapeamento cruzado aparece como uma ferramenta Que permite realizar comparações de forma sistematizada e passível de validação.

Entende-se como uma das principais possibilidades nesse contexto, a comparação de linguagens já utilizadas no cotidiano dos serviços com as terminologias oferecidas pelos sistemas de classificação e, desta forma, a identificação de lacunas Que podem existir em relação aos registros de enfermagem e também em relação às linguagens padronizadas, a ampliação das possibilidades de registro dos fenômenos Que são próprios da enfermagem, a criação de ligações entre diagnósticos ou problemas levantados pela enfermagem com resultados esperados e/ou intervenções, entre outros.

\section{CONSIDERAÇÕES FINAIS}

O presente estudo possibilitou identificar Que o mapeamento cruzado é uma ferramenta utilizada nas pesquisas da enfermagem sobre linguagens padronizadas, Que permite realizar comparações entre termos de forma sistematizada e passível de validação, incluindo a comparação entre as linguagens padronizadas com não padronizadas e entre linguagens padronizadas ou não padronizadas entre si.

Identificar e organizar os fenômenos Que fazem parte do domínio da enfermagem, obter uma maior efetividade nos registros realizados nos serviços, informatizar os dados e favorecer a inserção de dados em prontuários eletrônicos foram possibilidades relacionadas à utilização dos sistemas de classificação de enfermagem direta ou indiretamente apontadas nos estudos levantados.

Sem dúvida, estas são tendências atuais Que devem constituir foco de interesse da enfermagem brasileira Que já possui contribuições no cenário dos estudos sobre linguagens padronizadas com utilização do mapeamento cruzado.

Os resultados apontam para o interesse, em âmbito nacional e internacional, no estudo dos sistemas de classificação, o Que pode contribuir para o avanço do conhecimento dos fenômenos Que fazem parte do domínio da enfermagem e para o crescente reconhecimento desta disciplina no cenário científico mundial.

\section{REFERÊNCIAS}

I. Hyun S, Park HA. Cross-mapping the ICNP with NANDA, HHCC, Omaha System and NIC for unified nursing language system development. Int Nurs Rev 2002; 49(2): 99-1 10.

2. Moorhead S, Delaney C. Mapping nursing intervention data into the Nursing Interventions Classification (NIC): process and rules. Iowa City: Nurs Diagn 1997; 8(4): 137-44.

3. Lucena AF, Barros ALBL. Mapeamento cruzado: uma alternativa para a análise de dados em enfermagem. Acta Paul Enferm 2005; 18(1): 82-8.

4. Napoleão AA, Carvalho EC. Aplicabilidade de Intervenções Prioritárias da NIC para o Diagnóstico de Enfermagem Desobstrução Ineficaz de Vias Aéreas. Cogitare Enferm 2007; 12(1): 9-19.

5. Polit D, Beck CT, Hungler B. Fundamentos de pesquisa em enfermagem. Porto Alegre: Artmed; 2004.

6. Alburquerque, CC, Nóbrega, MML, Garcia, TR. Termos da linguagem de enfermagem identificados em registros de uma UTI neonatal. Rev Eletrônica Enferm 2006; 8(3): 336-48.

7. Lima MC, Araújo RTM, Trigueiro EV, Nóbrega MML, Garcia TR. Theoretical definitions of terms attributed to nursing phenomena identified in a school hospital. A systematic review Online Braz I Nurs 2007; 6.

8. Camiá GE, Barbieri M, Marin HF. Fenomenos de enfermagem identificados em consultas de planejamento familiar segundo a ICNP - Versão Beta 2. Rev Latino-Am Enfermagem 2006; 14(5): 674-81.
9. Coenen A, Ryan P, Sutton I Mapping nursing interventions from a hospital information system to the Nursing Interventions Classification (NIC). Nurs Diagn 1997; 8(4): 145-5 I.

10. Goossen WT, Ozbolt IG, Coenen A, Park HA, Mead C, Ehnfors $\mathrm{M}$, et al. Development of a provisional domain model for the nursing process for use within the Health Level 7 reference information model. I Am Med Inform Assoc 2004; I I (3): I 8694.

I I. Marin HF. Comparing nursing terms from patient records to the ICNP, beta version. Stud Health Technol Inform. 2002; 90: $232-5$.

12. Hyun S, Bakken S. Toward the creation of an ontology for nursing document sections: mapping section names to the LOINC semantic model. AMIA Annu Symp Proc 2006; 364-8.

13. Kuo $\mathrm{CH}$, Yen M. Cross-mapping ICNP terms with Taiwanese gynecological nursing records. I Nurs Res 2006; I4(4): 27 I8.

14. Zielstorff RD, Tronni C, Basque I, Griffin LR, Welebob EM. Mapping nursing diagnosis nomenclatures for coordinated care. Image I Nurs Sch 1998; 30(4): 369-73.

15. Chianca TC. Mapeando açoes de enfermagem da CIPESC para as intervenções da NIC. Rev Bras Enferm 2003; 56(5):5 I3-8.

16. Ehnfors M, Angermo LM, Berring L, Ehrenberg A, Lindhardt T, Rotegard AK, Thorell-Ekstrand I. Mapping VIPS concepts for nursing interventions to the ISO reference terminology model for nursing actions: A collaborative Scandinavian analysis. 
Stud Health Technol Inform 2006; 122:40 I-5.

17. Lee $\mathrm{E}$, Lee $\mathrm{M}$, Jung $\mathrm{OB}$. Mapping of nursing records into the NIC and the ICNP in a Korean oriental-medicine hospital. Comput Inform Nurs 2006; 24(6): 346-52.

18. Goossen W. Cross-mapping between three terminologies with the international standard nursing reference terminology model. Int I Nurs Terminol Classif 2006; 17(4): 153-64.

19. Park HT, Lu DF, Konicek D, Delaney C Nursing interventions classification in systematized nomenclature of medicine clinical terms: a cross-mapping validation. Comput Inform Nurs 2007; 25(4): 198-208.

20. Choi J, Jenkins ML, Cimino IJ, White TM, Bakken S. Toward semantic interoperability in home health care: formally representing OASIS items for integration into a conceptoriented terminology. J Am Med Inform Assoc 2005; 12(4): 410-7.

2 I. Lu DF, Park HT, Ucharattana P, Konicek D, Delaney C. Nursing outcomes classification in the systematized nomenclature of medicine clinical terms: a cross-mapping validation Comput Inform Nurs 2007; 25(3): 159-70.

22. Lu DF, Eichmann D, Konicek D, Park HT, Ucharattana P, Delaney C. Standardized nursing language in the systematized nomenclature of medicine clinical terms: A cross-mapping validation method. Comput Inform Nurs 2006; 24(5): 28896.

23. Delaney C, Moorhead S. Synthesis of methods, rules, and issues of standardizing nursing intervention language mapping. Nurs Diagn 1997; 8(4): 152-6.

24. Konig P, Siller M. Building a subset of ICNP terms for oncological patients. Stud Health Technol Inform 2006; 122: 900-I.

25. Nail LM, Lange LL. Using computerized clinical nursing data bases for nursing research. I Prof Nurs 1996; 12(4): $197-$ 206.

26. Goossen W.Testing the ISO nursing reference terminology model for mapping. Stud Health Technol Inform 2004; I 10 : 75-82.

27. Rotegard AK, Slaughter L, Ruland CM. Mapping nurses' natural language to oncology patients' symptom expressions. Stud Health Technol Inform 2006; 122: 987-8.

28. Bartz C, Coenen A, Hong WH. Participation in the International Classification for Nursing Practice (ICNP) programme. Stud Health Technol Inform 2006; 124: 157-61.

29. McCloskey IC, Bulechek GM. Classificação das Intervenções

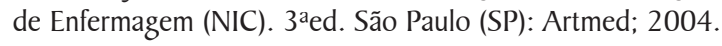

